\title{
An Electromechanical Valve for Solids $\dagger$
}

\author{
Mojtaba Ghadiri and Catherine M. Martin \\ Department of Chemical and Process Engineering, \\ University of Surrey *
}

\begin{abstract}
The operation and performance of a novel device, the Electromechanical Valve for Solids (EVS), for control of flow of granular materials are described.

The EVS applies a DC electric field to the granular material in a dense-phase state. This induces electrical interparticle forces which retard and can ultimately halt the flow, hence providing an actuating mechanism. The EVS has a very fast response time which makes it very useful for fast flow control. The response characteristics of the EVS and the results of flow control using a simple PID control action are reported.
\end{abstract}

\section{Introduction}

In many processes dealing with granular materials, the transfer of materials between various units of operation has often to take place at a prescribed rate. In such circumstances, the regulation and control of the flow is an important stage in processing and handling of granular materials. At present, this is accomplished by mechanical means such as a screw conveyor or a rotary star feeder, in which the material is translated forward at a rate depending on the angular speed of the screw or star. Although commonly used in industry for controlling the flow of granular materials, these devices have serious drawbacks which stem from the inherent features of their design and operation. For example, rotation of the screw against the stationary barrel causes attrition when handling delicate granules and erosion or seizure when hardling hard and abrasive materials. Also, the seal on the screw shaft, separating the system from the surrounding environment, is a fine component and may be easily damaged when handling dusty ma-

\footnotetext{
* Guildford GB-Surrey GU2 5XH, England

$\dagger$ Received May 21st, 1991
}

terials, especially at elevated pressures. Furthermore, these devices are slow in response, and have short term fluctuating characteristics and long term drift which make good flow control of processes with fast transients difficult.

The challenge faced in Particle Technology today is the development of new techniques for fast, reliable and accurate measurement and control of flow of particulate solids, as it has long been established for fluids. Recently some interesting 'non-mechanical' concepts have emerged, such as $\mathbf{L}, \mathbf{J}$ and $V$ valves based on aeration principles $[1,2]$, and the Magnetic Valve for Solids [3]. In the latter, the presence of a magnetizable powder under the influence of a magnetic field generates interparticle force. These forces lead to the formation of particle chains which bridge the gap between the electrodes, and hence impede the flow of solids.

It is well known that similar effects can be produced by the use of electric fields without any need for addition of special materials [4-8]. Consequently, in a recent development inspired by the above work, we have pursued the use of electric fields for controlling the flow of granular materials. It has been shown that the flow of dry granular materials in a dense phase form can be controlled by the 
watpplication of a DC field, provided a suitably designed set of electrodes is used [9]. This discovery has led to the development of the Electromechanical Valve for Solids(EVS)for both on/off operation and flow control of granular materials $[10]$. As in other non-mechanical valves, the EVS has no moving parts and is mounted in situ with no external mechanical connections so that it does not suffer from the problems associated with the mechanical devices as discussed above. It provides good flow control with a wide turndown ratio and very fast response time. It may also be used under adverse conditions such as elevated pressures and temperatures. The design of the electrodes forms an important feature for satisfactory operation of the valve. In this paper, various aspects of the design, principles of operation and performance of the EVS are described.

\section{Description of the device}

The EVS comprises essentially of a set of two electrodes which is installed within the duct in the path of the flowing material. The electrodes are connected externally to a high voltage supply(EHT)unit for establishing an electric field. Typical electrode configurations are shown in Fig. 1 for cylindrical and rectangular ducts. The upstream electrode may be in the form of a number of wires stretched across the duct or may simply consist of pins protruding into the duct. The downstream electrode may conveniently be in the form of a wire mesh or parallel wires fixed across the duct.

For the EVS to operate at all, it is essential that the flow in the space between the electrodes is in dense phase form. The presence of the electrodes obviously obstructs the flow to some extent. Observations of particle motion in the space between the electrodes in a narrow rectangular column, providing essentially a two-dimensional flow, indicate that as soon as the flow is transformed into the lean phase in this region, the actuating mechanism is lost completely. Observations of particle motion in the region of the electrodes also indicate that halt-
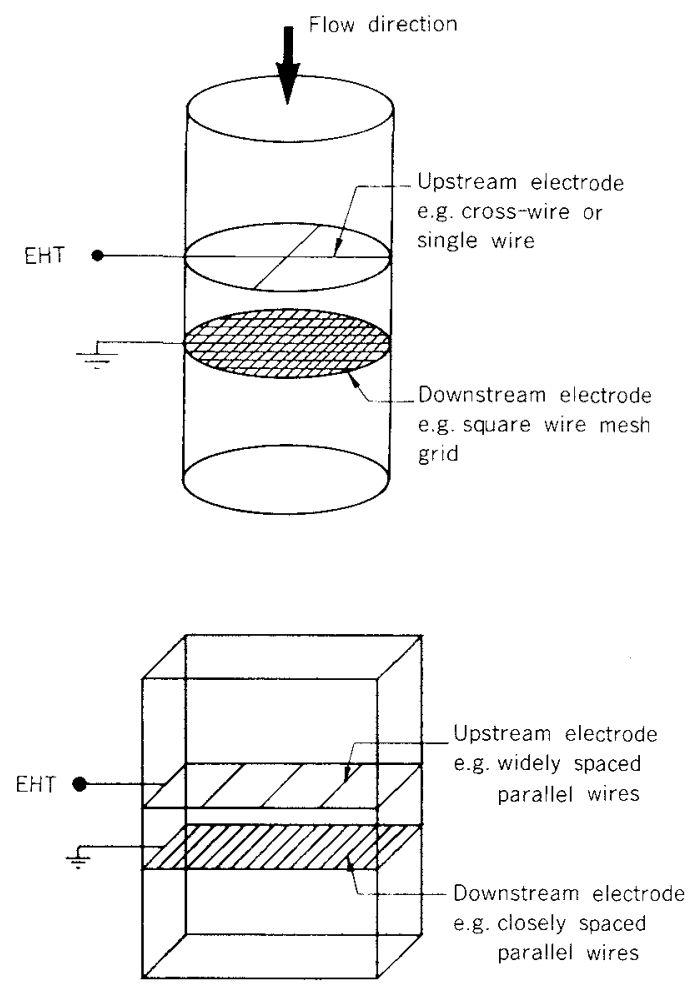

Fig. 1 Typical clectrode configurations of the EVS in cylindrical and rectangular ducts.

ing of flow on application of the electric field is brought about by arching of particles over the mesh openings of the downstream electrode. Consequently, the key features in the design of the electrodes are that the geometry of the electrodes should be such that(a)the upstream electrode does not obstruct the dense phase flow of particles to an extent which could transform the flow into the lean phase form in the space between the electrodes, thus losing continuous interparticle and particle-electrode contacts:(b)the downstream electrode has sufficiently small openings so as to enable impeding and ultimately halting of the flow by the application of a suitably high electric field. To satisfy condition(b), there is usually some impeding of the flow by the downstream electrode even in the absence of an electric field, so that the flow past this electrode is always tranformed into the lean phase form. Consequently, the use of more than two electrodes is deleterious to the stability of the operation of the EVS.

The feasibility of the concept of flow 
pontrat of granular materials by the application of an electric field has been demonstrated in two cylindrical columns of 64 and $115 \mathrm{~mm}$ I.D., and in a rectangular narrow slot of $200 \mathrm{~mm} \times 12 \mathrm{~mm}$. A large number of materials have been tested so far including sand, coal, salt, FCC catalyst powder, seeds, spray dried powders, and various chemicals and foodstuff. As the action of the EVS is to impede the flow of free flowing materials, for very cohesive materials discharge aids such as aeration or vibration may be needed. Once the material is made to flow, the EVS performs

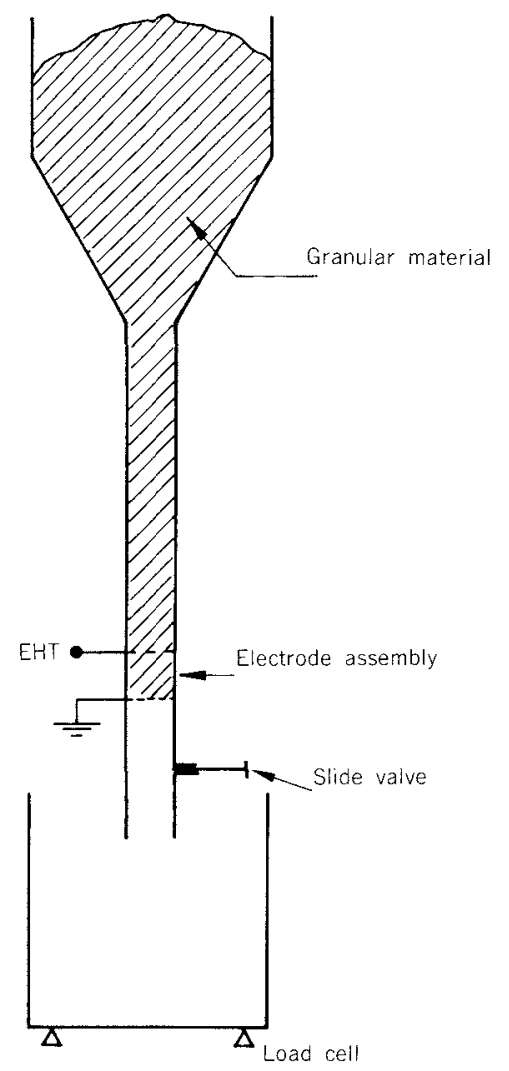

Fig.2 Schematic diagram of test rig for measuring flowrate through EVS.

just as for non-cohesive materials, as is described below.

Figure 2 shows a schematic diagram of the test rig incorporating the electrode assembly. A slide or a flap door is needed downstream of the electrodes. It is initially closed when filling the column and the storage bin, but is left open thereafter as the bed of material can be supported by the electric field.
If a continuous field is applied, a steady flowrate is obtained which is a function of the field magnitude. Alternatively, the electric field may be pulsed, where the frequency, pulse width and amplitude may all be varied to control the flow. The performance of

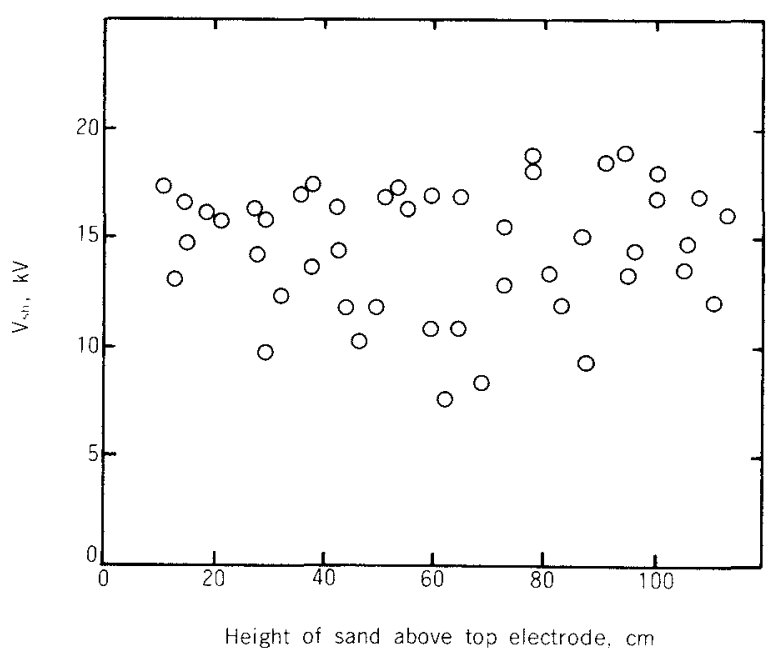

Fig.3 Effect of height of material on static holding voltage for $600-1200, \mu \mathrm{m}$ sand in $115 \mathrm{~mm}$ column. Upper electrode: cross-wire: lower electrode mesh opening: $10 \mathrm{~mm}$ : inter-electrode gap (IEG): $36 \mathrm{~mm}$.

these two modes of operation is discussed below.

\subsection{Continuous DC Field}

If an electric field is first set up to a suitably high level before opening the flap door, the particles between the electrodes will be frozen', thus supporting the material above the electrodes. No particles will flow when the door is opened except those in the space between the lower electrode and the flap door. If the voltage applied to the electrodes is then gradually reduced, a point is reached when the material begins to flow. This is referred to as the threshold of the static holding voltage, $\mathrm{V}_{\mathrm{th}}$, which is found to be effectively independent of the height of material above the upstream electrode, but scattered within a range. A typical example is shown in Fig. $\mathbf{3}$ for sand. The scatter is observed for all materials and electrode configurations tested so far, 
Wand is believed to be due to variations in the packing structure, which depend on the filling history, and possible switching between the active and passive states on the initiation of flow. The upper limit of $\mathrm{V}_{\mathrm{sh}}$ is of interest here as it represents the maximum possible potential required to prevent the granular material flowing from a static state. Typical values of $V_{\mathrm{sh}}$ for several materials and electrode configurations are given in Table $\mathbf{1}$.
If the voltage is reduced to a value which allows the material to flow, a steady-state flowrate is attained which is a function of the applied voltage. The flowrates are obtained from the gradient of the weight versus time traces recorded by a load cell from which the collection vessel is suspended. The performances of the EVS for the cylindrical columns of diameter 64 and $115 \mathrm{~mm}$ are shown in Fig.4 for sand in the size range 600-1200 $\mu$

Table 1 Upper limits of $V_{t h}$ for materials in varying electrode configurations.

\begin{tabular}{|c|c|c|c|c|c|}
\hline Material & $\begin{array}{c}\text { Column shape and size } \\
(\mathrm{mm})\end{array}$ & $\begin{array}{c}\text { Lower electrode openings } \\
(\mathrm{mm})\end{array}$ & $\begin{array}{l}\text { Inter-electrode gap (IEG) } \\
(\mathrm{mm})\end{array}$ & $\begin{array}{c}\text { Particle size } \\
(\mathrm{mm})\end{array}$ & $\begin{array}{c}V_{\text {w }} \\
(\mathrm{kV}) \\
\end{array}$ \\
\hline \multirow{8}{*}{ Sand } & \multirow{4}{*}{ Cyl. 64} & \multirow{2}{*}{ Mesh, 5} & \multirow{2}{*}{36} & $0.3-0.6$ & 11 \\
\hline & & & & $0.6-1.2$ & 10 \\
\hline & & \multirow{2}{*}{ Mesh. 10} & \multirow{2}{*}{36} & $0.6-1.2$ & 17 \\
\hline & & & & $1.2-2.4$ & 15 \\
\hline & \multirow{4}{*}{ Cyl. 115} & \multirow{2}{*}{ Mesh, 5} & \multirow{2}{*}{36} & $0.3-0.6$ & 18 \\
\hline & & & & $0.6-1.2$ & 18 \\
\hline & & \multirow{2}{*}{ Mesh, 10} & \multirow{2}{*}{36} & $0.6-1.2$ & 19 \\
\hline & & & & $1.2-2.4$ & 17 \\
\hline \multirow{2}{*}{ Coal } & Cyl, 64 & Mesh, 10 & 36 & $0.3-1.4$ & 4 \\
\hline & Cyl, 115 & Mesh. 10 & 36 & $0.6-1.2$ & 11 \\
\hline $\mathrm{Coal} / \mathrm{Sand} 50 / 50 \mathrm{wt}^{9} \%$ & Cyl, 115 & Mesh, 10 & 36 & $0.6-1.2$ & 14 \\
\hline \multirow{2}{*}{$\mathrm{NaCl}$ salt } & Cyl, 64 & Mesh. 5 & 20 & $0.35-0.6$ & 10 \\
\hline & Rect & Slot. 5 & 20 & $0.35-0.6$ & 12 \\
\hline Granulated sugar & Rect & Mesh, 3 & 20 & $0.18-1.7$ & 5 \\
\hline Turnip seeds & Rect & Slot. 5 & 20 & $1.2-1.7$ & 3.5 \\
\hline Soap powder & Rect & Slot. 5 & 20 & $0.12-1.4$ & 5 \\
\hline MgO powder & Rect & Mesh. I.5 & 20 & $0.04-0.35$ & 17 \\
\hline FCC powder & Rect & Mesh. 1 & 20 & $0.05-0.10$ & 2 \\
\hline
\end{tabular}

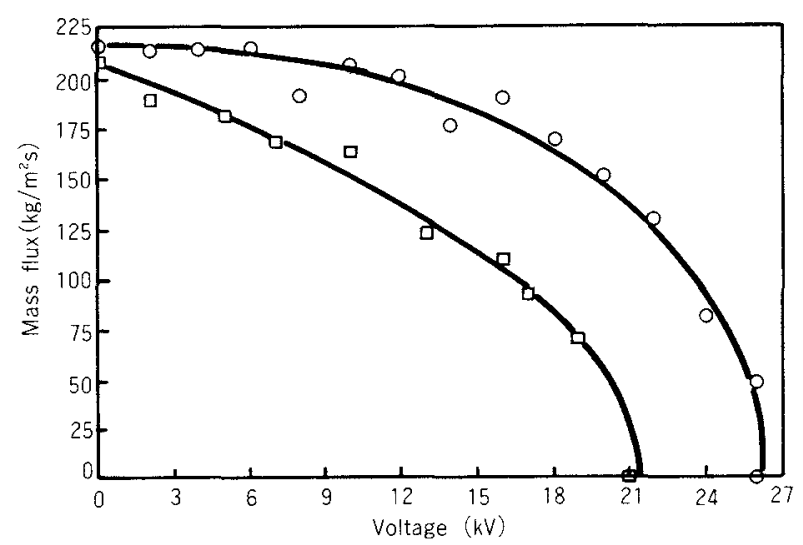

Fig.4 Effect of voltage on mass flux in cylindrical column for 600-1200 $\mu \mathrm{m}$ sand. Upper electrode: cross-wire; lower electrode mesh opening: $10 \mathrm{~mm}$; IEG: $36 \mathrm{~mm}$. Column diameter: $0,115 \mathrm{~mm}$; $\square$, $64 \mathrm{~mm}$. Best fit from flow model equation(3):- m. The electrode configuration shown in Fig.1 was used here with $10 \mathrm{~mm}$ square openings in the downstream electrode and an interelectrode gap (IEG) of $36 \mathrm{~mm}$. The performance of the EVS for the rectangular column is shown in Fig.5 for sand and glass ballotini both having a particle size range of $600-850 \mu \mathrm{m}$. In this case, the downstream electrode consisted of parallel wires extending across the duct as shown in Fig.1, with a spacing of $5 \mathrm{~mm}$ between the wires, and the upstream electrode consisted of pins extending 1-2 $\mathrm{mm}$ into the bed at intervals of 10 $\mathrm{mm}$ along the front and back faces of the column. The curves shown in Figs. 4 and $\mathbf{5}$ were obtained by fitting the experimental data to a semi-empirical flow model, equation (3), 


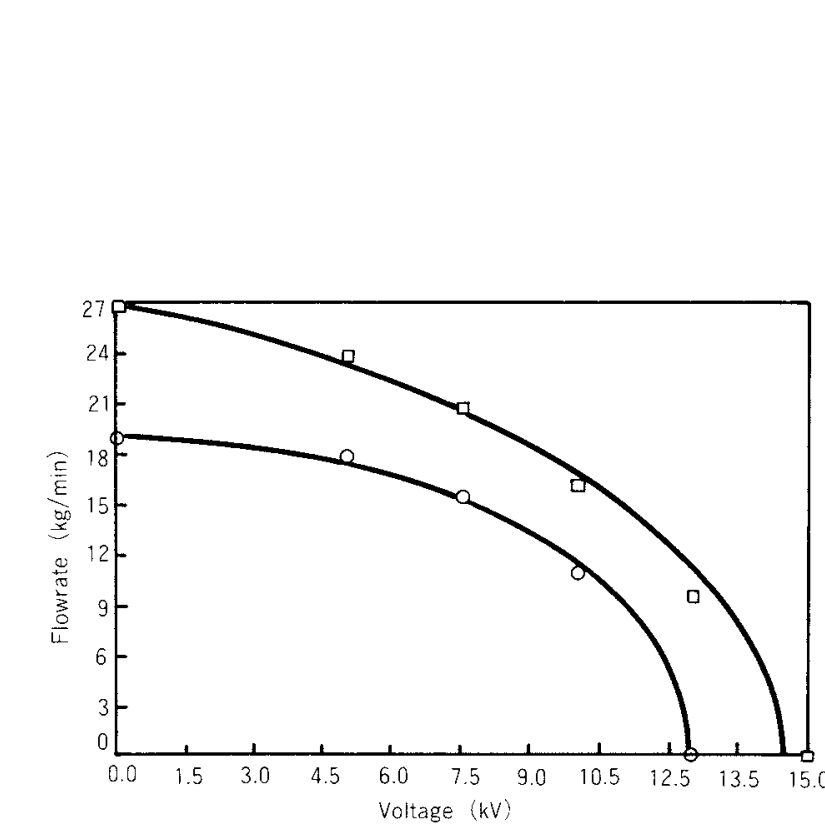

Fig.5 Effect of voltage on flowrate in rectangular column. Upper electrode: pins; lower electrode slot width: $5 \mathrm{~mm}$; IEG: $20 \mathrm{~mm}$. 0 :sand $(600-850$ $\mu \mathrm{m}) ; \square$ : glass ballotini $(600-850 \mu \mathrm{m})$. Best fit from flow model equation( 3 ):

which is discussed below. Figures 4 and $\mathbf{5}$ show that similar flow profiles are obtained in the cylindrical and rectangular columns. The results therefore show a common trend for the continuous DC field. The flowrate curves have a flat almost horizontal section at low voltages, followed by a nearly linear section at higher voltages. As the flowrate decreases to below about $50 \%$ of the full flow, the curves become quite steep and the flowrate drops off quickly to zero. Consequently, for good flow control using a continuous field, only that portion of the curve between full flow and about $50 \%$ of the full flow may be used. The low flowrate range is best handled by operating in the pulsating field mode, as is discussed below.

The current through the Valve presents an Ohmic relation with the applied voltage. For the results shown in Fig. 4 for sand, the current was about $25 \mu \mathrm{A}$ at $25 \mathrm{kV}$, giving a remarkably low power consumption of $0.625 \mathrm{~W}$.

\subsection{Pulsating DC Field}

In this mode, the pulse amplitude, frequency and width can all be varied in order to obtain a desired flow rate. The effects of varying the width and frequency of the pulse are shown in Figs.6 and 7, respectively, for different materials. In these tests, the pulse amplitude was set at a sufficiently high value

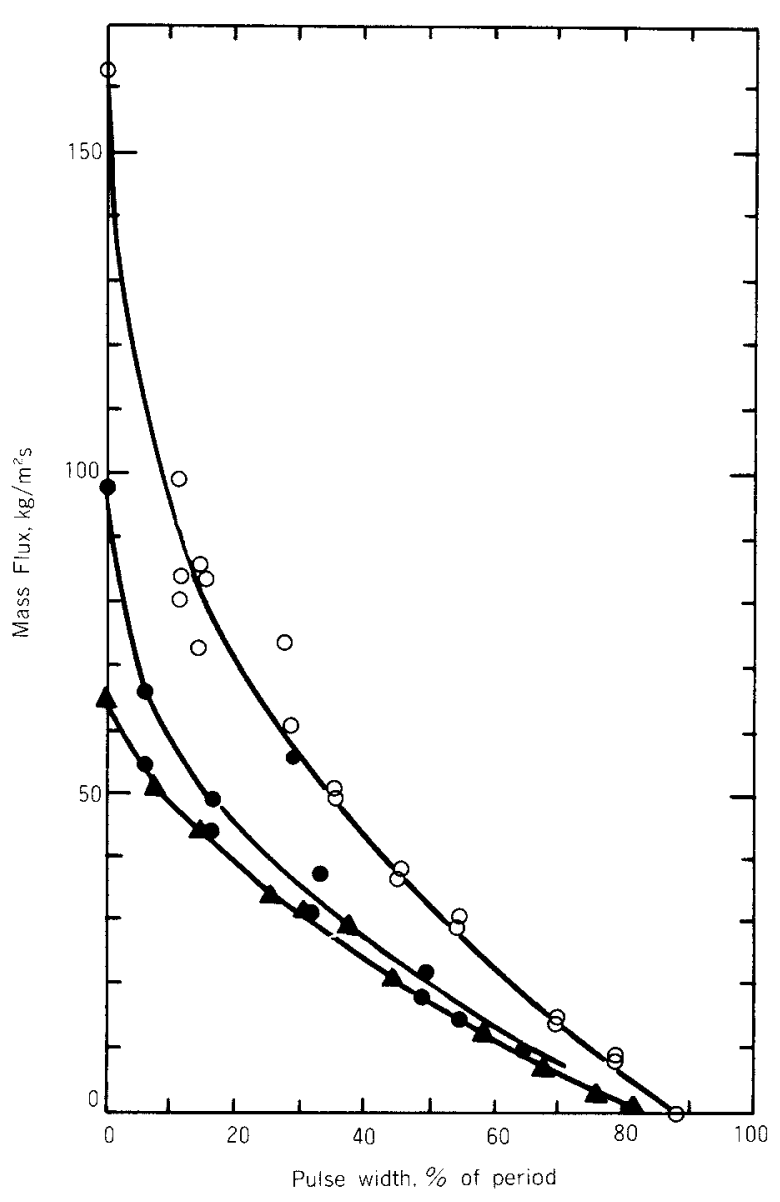

Fig.6 Effect of pulse width on mass flux for pulsating field operation. Pulse frequency $=1.5 \mathrm{~Hz}$; pulse amplitude $=28 \mathrm{kV}$, particle diameter $=600-1200$ $\mu \mathrm{m}$; column diameter $=64 \mathrm{~mm}$; upper electrode $=$ cross-wire; lower electrode mesh opening $=10$ $\mathrm{mm}$ square; $\mathrm{IEG}=36 \mathrm{~mm}$. $\mathbf{A}$ : coal; $\mathrm{O}$ : sand; $\mathrm{coal} / \operatorname{sand}(50 / 50 \mathrm{wt} \%)$.

in order to achieve fast and complete halting of the flow on application of a pulse. The data shown in Fig.6 were obtained using the $64 \mathrm{~mm}$ diameter column with the electrode configuration shown in Fig1. The frequency response shown in Fig.7 was obtained using the narrow rectangular duct, with the upstream electrode consisting of the pin arrangement as described above, and the downstream electrode consisting of parallel wires forming slots of $5 \mathrm{~mm}$ width.

The results in Figs. $\mathbf{6}$ and 7 show a trend opposite to that of the continuous field. For high flowrates, the curves are steep, providing poor control, whereas for small flowrates the curves 


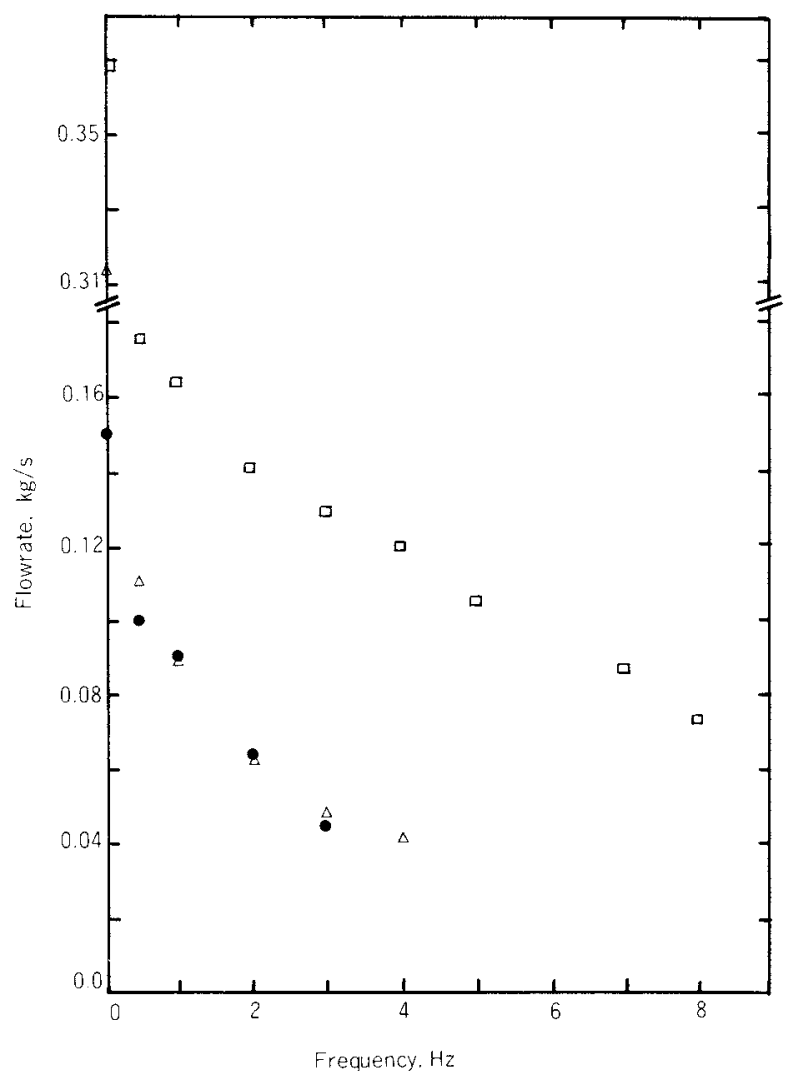

Fig.7 Effect of pulse frequency on flowrate for pulsating field operation in rectangular column. Pulse width $=50 \%$ of period. Upper electrode $1-2 \mathrm{~mm}$ long pins spaced $10 \mathrm{~mm}$ apart; lower electrode - slots of $5 \mathrm{~mm}$ width; IEG $=20 \mathrm{~mm}$. turnip seeds (1.2-1.7 mm), pulse amplitude $=3.5$ $k V ; \triangle$ : sand $(0.6-0.85 \mathrm{~mm})$, pulse amplitude $=11$ $k V$; $:$ salt $(0.35-0.6 \mathrm{~mm})$, pulse amplitude -12 kV.

become nearly linear with a small slope. Thus the two modes of operation complement each other to provide an overall wide turndown ratio for practical situations.

In Fig.7, the maximum frequency tested with each material was limited by the relatively long charging and discharging times of the overall circuit. This is because the field was pulsed by applying an inhibiting pulse to the low voltage input of the EHT unit, and therefore the EHT's capacitor had to be charged again each time the field was switched on and then discharged through the material when the field was switched off. The bulk resistivity of the material is therefore an important factor influencing the discharge time. The electrical properties of the materials tested here are given in Table 2, as measured in situ between the electrodes in the rectangular column using a Wayne Kerr electrical bridge. The $\mathrm{NaCl}$ salt had a much shorter discharge time than the sand or turnip seeds, due to its lower resistivity. It was therefore possible to pulse at higher frequencies with the salt.

The overall circuit response therefore represents the combined responses of the EHT unit, charging/discharging through the bed of material and particle movement in the region of the electrodes. The maximum frequency is at present limited mainly by discharging a large capacitor through the material. Much

Table 2 Electrical properties of materials as meatsured in situ across electrodes in rectangular column.

\begin{tabular}{lccc}
\hline Material & $\begin{array}{c}\text { Particle size } \\
(\mathrm{mm})\end{array}$ & $\begin{array}{c}\text { Resistance } \\
(\mathrm{M} \Omega)\end{array}$ & $\begin{array}{c}\text { Capacitance } \\
(\mathrm{pF})\end{array}$ \\
\hline $\begin{array}{l}\text { Turnip } \\
\text { seeds }\end{array}$ & $1.27-1.7$ & 48 & 15 \\
$\begin{array}{l}\text { Sand } \\
\mathrm{NaCl} \text { salt } \\
\text { (without anti- }\end{array}$ & $0.6-0.85$ & 43 & 12 \\
caking agent) & $0.35-0.6$ & 14 & 14 \\
\hline
\end{tabular}

higher frequencies will be possible by switching the electric field at the high voltage output of the EHT unit, and shorting the electrodes, thus avoiding slow discharge of the EHT's capacitor through the material. The absolute upper limit of frequency depends on the charge relaxation time of the particles which is typically in the order of milliseconds. The development of such a high voltage switch is currently in hand.

\subsection{Closed loop flow control with the EVS}

The potential of the EVS for setpoint flow control in a closed loop system has so far been investigated only for the continuous field mode of operation [11]. The test rig shown in Fig.2 was used for disparsing $\mathrm{NaCl}$ salt (90-600) $\mu \mathrm{m}$ diameter from the $64 \mathrm{~mm}$ column with the electrode configuration shown in Fig.1. The gap between the electrodes was $20 \mathrm{~mm}$ and the downstream electrode 
pperation was carried out by a programme developed in-house using a Sinclair QL computer which was interfaced with the EHT for controlling the voltage, and with a tension load cell for monitoring the flowrate.

In order to exploit the fast response time of the EVS for fast control, it is necessary to use an equally fast sampling rate to monitor the instantaneous flowrate. In the system used here, the flowrate was calculated from the change in accumulated weight measured by the load cell over the sampling interval. Since the load cell had a continuous analog output which was digitised at the computer interface by a fast 16 bit $\mathrm{A} / \mathrm{D}$ converter, it was possible, in principal, to use very fast sampling rates for monitoring the flowrate. However, it was found that the variability or 'noise' in the flowrate readings increased significantly with increasing sampling rate. This is illustrated in Fig.8, where the relative standard deviation calculated from twenty consecutive flowrate readings is shown for varying sampling intervals. Figure 8 shows that it is necessary to use a sampling interval of about $1.0 \mathrm{~s}$ to reduce the relative standard deviation to 10 per cent. Therefore, although the load cell is capable of much faster output frequencies, precise and accurate measurements can only be obtained at frequencies less than about $1 \mathrm{~Hz}$. This response results from the response of the load cell and the transportation time of the material from the electrodes to the weighing bin. For the experimental setup used here, where the height between the EVS and the bottom of the bin is about $1.0 \mathrm{~m}$, the transportation time has a mimimum value of about $0.4 \mathrm{~s}$, as estimated by the free fall of the particles.

Based on the results in Fig.8, a frequency of $1 \mathrm{~Hz}$ was used initially to monitor the flowrate through the EVS for the PID control operation. However, it became immediately evident that while $1 \mathrm{~Hz}$ was sufficiently slow to produce stable measurements for steady-state flow, an unacceptable amount of instability was produced in the case of transient flows produced by controlling to a setpoint. It was therefore necessary to base the

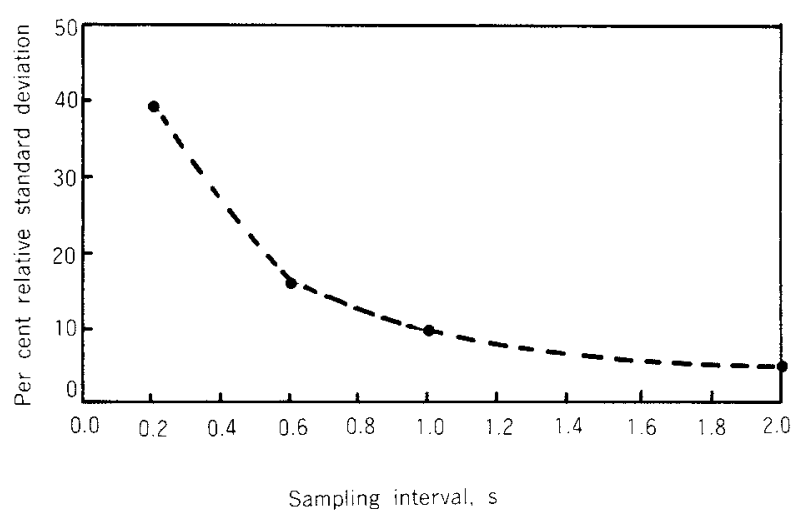

Fig.8 Effect of sampling interval on per cent relative standard deviation for flowrates measured using load cell interfaced with Sinclair QL computer.

PID control on a moving average flowrate calculated from five flowrate measurements at one second intervals. Using a moving average, to represent the instantaneous' flowrate, slowed down the system response significantly. However, using the moving average method at least allowed the response limitations of the load cell to be overcome in such a way that the control abilities of the EVS could be observed.

The response of the EVS to three changes in flowrate setpoint using a constant gain of $0.0678 \mathrm{~kg} \mathrm{~s}^{-1} \mathrm{k} \mathrm{V}^{-1}$ is shown in Fig.9. While the response is slow, the final setpoint error is negligible. For effective control, a PID

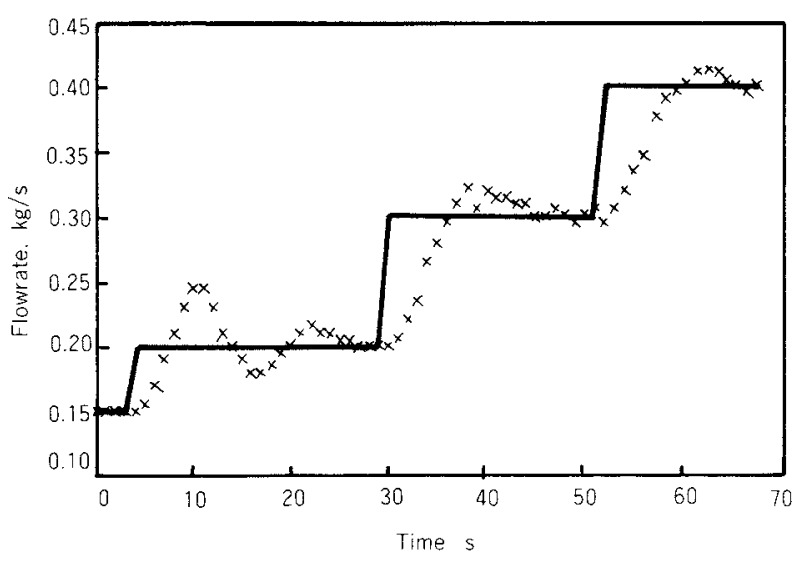

Fig.9 Response of EVS to PID control for changing setpoint with $\mathrm{NaCl}$ salt $(90-600 \mu \mathrm{m})$ in $64 \mathrm{~mm}$ column with $5 \mathrm{~mm}$ mesh openings. Flowrate setpoint:-_; measured moving average flowrate response: $\mathrm{X}$. 
Wmethod with constant gain is really only suited to linear processes. As shown by the performance curves in Fig.4 and $\mathbf{5}$, the response of the EVS is not linear over the full flowrate range. However, by taking a limited flowrate range over the full curve for control with constant again, the nonlinearity is small and acceptable control is obtained. The gain used for control in Fig.9 was determined by trial and error tuning for a flowrate range of 0.15 to $0.45 \mathrm{~kg} \mathrm{~s}^{-1}$ where $0.45 \mathrm{~kg} \mathrm{~s}^{-1}$ was the maximum flowrate when zero field was applied. The setpoint of $0.2 \mathrm{~kg} \mathrm{~s}^{-1}$ is at the low flowrate end of the full range, and therefore lies on the steep part of the valve performance curve, where the flowrate is very sensitive to any change in voltage. Therefore, the response of the EVS in Fig.9 for the setpoint of $0.2 \mathrm{~kg}$ $\mathrm{S}^{-1}$ shows significant oscillation. The setpoints of 0.3 and $0.4 \mathrm{~kg} \mathrm{~s}^{-1}$ move progressively onto the flatter part of the valve performance curve, so that the oscillating response of the EVS to these setpoints become damped in Fig.9. Therefore, an obvious improvement to this control method would be the introduction of a self-tuning gain which is updated according to the flowrate response in the range of the the setpoint.

\section{Analysis}

The actuating mechanism which is considered to be responsible for the action of the EVS depends on the generation of electroclamping forces by a small electric current flowing over the particle surfaces in the region of the electrodes [12]. The electroclamping forces stem from the very nature of the material, i.e. being in particulate form. Individual particles are in point contact' with each other at rest or when they are moving in a dense phase form. When an electric field is set up across two electrodes inserted in a bed of particles, there will be some charge leakage through the bed via these contact points, provided that the particles are not too conductive so as to draw excessively high corrents, nor too resistive so as to draw such low current that there is negligible effect. Because the current path is constricted in the region of the contactpoint, the electric field in the gap surrounding the contact area is greatly enhanced. Depending on the size and shape of the contact area, this field is highly non-uniform and can be several orders of magnitude larger than the average field. This mechanism has been analysed by McLean [4], Dietz and Melcher [5]. and more recently and comprehensively by Moslehi and Self [6] to describe the adhesion of the precipitated dust layer in the electrostatic precipitators, and various effects in electro-fluidised and packed beds. The attractive forces between the particles resulting from this phenomenon are very high, and comparable in magnitude with other external forces such as those due to gravity, as in the case of the EVS, and those due to drag resulting from pressure drop in electrically enhanced fabric filters [13].

There are several theoretical models of the electroclamping force [4-6]. These models differ in details, depending on the mechanical properties of the contact points, and on the limit of electric field breakdown in the interparticle gap. However, they all relate the clamping force to the average applied electric field strength, $E_{a}$, for a single contact between two spheres, or between a sphere and a conductive plane, and can be represented by:

$\mathrm{f}_{\mathrm{e}}=\alpha \mathrm{E}_{\mathrm{a}}$

where $f_{c}$ is the interparticle electroclamping force, $\alpha$ is a function of the physical and electrical properties of the particles, and $\beta$ is the power law index. The value of $\beta$ depends on the characteristics of the contact point, i.e. its initial size and deformation under loading. For non-deforming contact points, $\beta$ is analytically calculated and is equal to $2[4]$. However, there is usually some elastic deformation resulting from the electrical force alone, or from a combination of electrical and mechanical forces, and this leads to a less enhanced field in the gap surrounding the contact point, and hence to a lower power dependence. The models differ in the value of $\beta$, but they all fall within the range 1.2 to 1.5 $[4,6]$. In all these models, the elastic deformation due to the electric field is 
described by the Hertz analysis. If the size of the contact point is very small, such as for very fine particles or for particles with sharp asperities, then the electric field could exceed the limit of breakdown; in this case $\beta$ reduces to unity [5]. Recently it has been shown that the Hertz analysis may not adequately describe the pattern of contact deformation under an electric field [12], so that the formulation of the models of the electroclamping force for a single contact may be in error. However, in the analysis of the macroscopic flow behaviour presented below, only a general analogy with equation(1) is made, without taking any prescribed value for the power index.

The geometry and configuration of the electrodes used here are such that the electroc lamping force can produce counteracting compressive stresses as well as a net upward force. The increased compressive stresses lead to higher shear stresses on the electrodes and on the walls, thus providing a means to support the weight of the bed. Indeed it is found that roughening the walls in the space between the electrodes results in a reduction in $V_{\mathrm{sh}}$. The net upward force on the other hand can act to support the stable arches which form over the openings of the downstream electrode on halting of the flow. However, because of the complex geometry of the electrodes, a mathematical analysis of the mechanics of arching in the presence of these forces is somewhat involved. Consequently, a simpler geometry, i.e. that of a wedge on which planar electrodes have been installed, has so far been attempted. The results of this analysis are presented elsewhere [14], where the arch span has been related to the electric field strengh.

To model the effect of the electric field on flow, an analogy has been made with the effect of interstitial gas pressure gradients on discharge of fine granular materials from hoppers and bins. Nedderman et al. [15] have shown that the Beverloo correlation could be modified to account for the effect of air drag on the flowrate such that
$\mathrm{W} \propto \mathrm{g}^{1 / 2}\left(1+\left.\frac{1}{\partial \rho} \frac{\mathrm{dP}}{\mathrm{dr}}\right|_{\mathrm{r}=\mathrm{r}_{0}}\right)^{1 / 2}$

where $P$ is the interstitial pressure, $r$ is the radial coordinate in the conical hopper, and $r_{0}$ is the value of $r$ at the orifice.

According to equation(2), the flow due to gravity is reduced by the gradient of the interstitial gas pressure at the orifice, which provides a net external force opposite to the gravitational force. In the case where an electric field is applied, the 'drag' force is considered to result from the electrical forces impeding the flow. Therefore as a first approximation, it is proposed that the drag effect caused by the field may take a similar functional form to that of equation(1), such that the following relationship describes the flowrate response to the applied field:

$\mathrm{W}=\mathrm{W}_{\circ}\left(1-\lambda \mathrm{E}_{\mathrm{a}}^{\gamma}\right)^{1 / 2}$

where $\lambda$ and $\gamma$ are constants, and $W_{0}$ is the flowrate without an applied field. For convenience in fitting parameters $\lambda$ and $\gamma$ to the experimental data, equation(3)has been rearranged such that

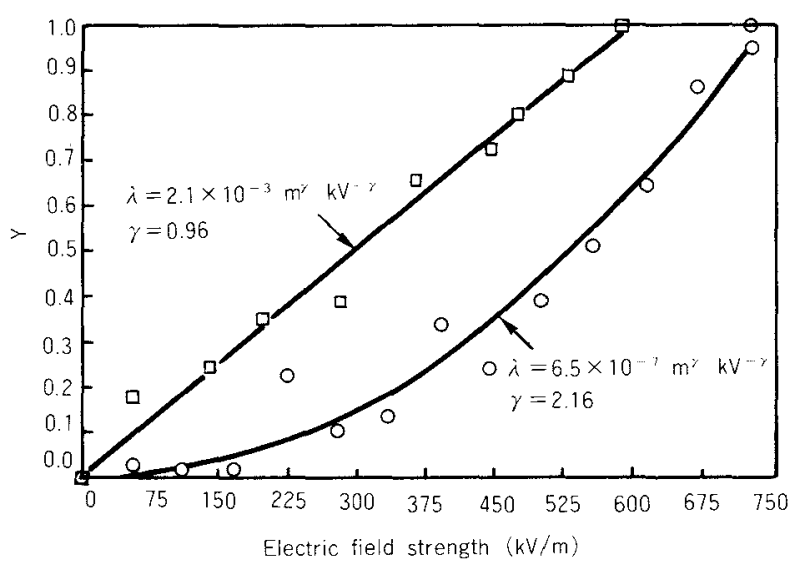

Fig.10 Fit of flow model, equation(4), to expermental data from Fig.4 for cylindrical column. Experimentals: $0,115 \mathrm{~mm} ; \square, 64 \mathrm{~mm}$ Best fit from model:-- -

$\mathrm{Y}=\lambda \mathrm{E}_{\mathrm{a}}^{\gamma}$

Equation(4)has been fit to a large set of experimental data for $\mathrm{W}, \mathrm{W}_{0}$ and $\mathrm{E}_{\mathrm{i}}$, collected from both the cylindrical and rectangular 


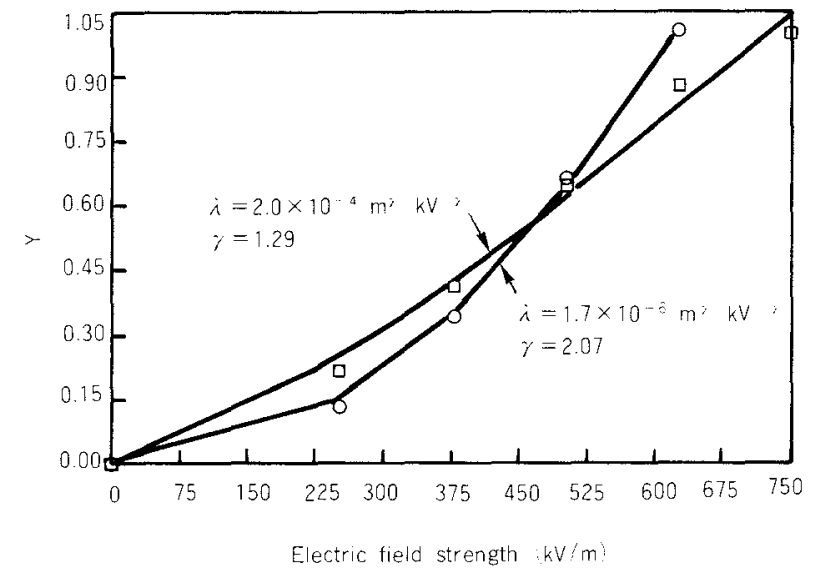

Fig.11 Fit of flow model, equation(4), to experimental date from Fig.5 for rectangular column. Experimental:O, sand; $\square$, grass ballotini. Best fit from model:-

columns, using a non-linear least squares routine to obtain values of $\lambda$ and $\gamma$. In Figs. 10 and 11, the experimental values of $Y$ are compared to the best fit values from equation (4)for the same data shown in Figs.4 and 5. From Figs.10 and $\mathbf{1 1}$, it is clear that very good fits are obtained for individual data sets. However, when all the data sets are compared, the values of $\lambda$ and $\gamma$ cover wide ranges, with no apparent trends related to the experimental conditions. Therefore, while it seems that the flow model given by equation(4)is suitable for calibrating the EVS for practical operation, the formulation does not present a verifiable description of the process based on a fundamental mechanistic analysis, as is necessary for effective optimisation of the EVS and for application of the results to other processes where electric fields are applied to particulate solids. Therefore, in future, it is necessary to proceed with a more rigorous analysis, where the precisely defined electrical and mechanical stress distributions are incorporated into the macroscopic constitutive equations of motion. Indeed, the approach outlined above for modelling the steady-state flow in the EVS is a simplistic one, since the average value of the electric field is used in the analyses, without considering the effect of the highly non-uniform fields which are produced in the complex electrode configurations used here [10]. A model for the static holding voltage, $V_{\mathrm{s}}$, has been developed elsewhere using a well defined 2-dimensional field configuration, thus allowing a much more rigorous analysis [14]. This approach is obviously also necessary for successful modelling of the steady-state flow.

\section{Conclusions}

The EVS has considerable potential for use in a variety of bulk solids processes. In on/off operation, the EVS can be used for dosing and fast packaging. For controlling a continuously flowing material in a metering or feeding process, the EVS can achieve a wide turndown ratio by using a continuously applied voltage for large flowrates and a pulsating voltage for small flowrates. Using a simple PID control programme in a computerised closed loop system, the EVS produced good flowrate control with respect to final setpoint error, but the fast response of the EVS could not be exploited due to the limitations of the load cell for measuring the flowrate and due to the long transportation time.

For flow with a continuously applied field, a semi-empirical correlation is presented to describe the steady-state flowrate through the EVS as a function of the field strength. The correlation provides a good fit to the data for calibration purposes. However, the fitted parameters from the correlation do not appear to follow any trends. Therefore, a more rigorous mechanistic analysis of the interacting forces in the presence of nonuniform fields is necessary in order to develop a flow model which will be effective for design and optimization of the EVS.

Overall, the EVS offers many advantages over conventional mechanical valves for controlling the flow of granular materials. It has no moving parts and so wear of the material and the valve itself is reduced. In addition, the power consumption is remarkably low. Therefore, the EVS offers potential savings in both operating and capital costs. Most importantly, the EVS has a very fast response time which makes it very useful for fast flow control. 


\section{Acknowiledgments}

The development of the EVS was made possible by financial support from the Research Committee and the Technology Transfer Committee of the University of Surrey. Further research on the electromechanics of the valve is being continued with support from the Science and Engineering Research Council. The authors should express their gratitude to Prof. R. Clift and Dr. U. Tüzuin for their interest in this work and their contributions to stimulating discussions.

\section{Nomenclature}

$E_{\mathrm{a}}=$ applied electric field, $\mathrm{V} \mathrm{\textrm {m } ^ { - 1 }}$

$f_{\mathrm{e}}=$ single contact electroclamping force, $\mathrm{N}$

$\mathrm{g}=$ gravitational acceleration, $\mathrm{m} \mathrm{s}^{-2}$

$\mathrm{P}=$ interstitial gas pressure, $\mathrm{Pa}$

$\mathrm{r}=$ radial position in conical hopper; $=\mathrm{r}_{0}$ at orifice, $\mathrm{m}$

$V_{\mathrm{s}}=$ static holding voltage, $\mathrm{V}$

$\mathrm{W}=$ mass flowrate, $\mathrm{kg} \mathrm{s}^{-1}$

$\mathrm{W}_{0}=$ mass flowrate through electrodes without electric field, $\mathrm{kg} \mathrm{s}^{-1}$

$\mathrm{Y}=$ parameter defined in flow model equation(4)

$a=$ parameter in electroclamping force models, NV " n $\mathrm{n}$

$\beta=$ exponent in electroclamping force models

$\gamma=$ parameter in electrically-modified flow model equation(4)

$\lambda=$ parameter in electrically-modified flow model equation(4), $\mathrm{V}^{\prime \prime} \mathrm{m}^{\prime}$

$\rho=$ bulk material flowing density, $\mathrm{kg} \mathrm{m}^{-3}$

\section{References}

1) T.M. Knowlton, in "Gas Fluidization Technology", Ed. D.G. Geldart, Ch. 12, p.341, John Wiley \& Sons. Chichester, 1986.

$2)$ L.S. Leung, Y.O. Chong and J. Lottes, Powder Technol., 49 (1987)271.

$3)$ E.Jaraiz-M., O. Levenspiel and T.J. Fitzgerald. Chem.Eng.Sci., 38(1983)107.

4) K.J. McLean, J.A.P.C.A. 27 (1977)1100.

5) P.W. Dietz and J.R.Melcher, A.I.Ch.E.Symposium Series, 74 No. 175(1978)166

6) G.B. Moslehi and S.A. Self, IEEE Trans.Ind.Appl., IA-20, No.6(1984)1598.

7) H.A. Pohl, Dielectrophoresis, Cambridge University Press, Cambridge, 1978.

8) T.B. Jones, Journal of Electrostatics, 6 (1979), 69.

9) M. Ghadiri and R. Clift. European Patent Application No. 87308304. 2. 1987.

10) M. Ghadiri, C.M. Martin and J. E.P. Morgan, submitteditted to Powder Technol. (1991).

11) C.M. Martin and M. Ghadiri, BULK 2000: Bulk Materials Handling - Towards the Year 2000, London, UK, 29-31 October 1991.

12) C.M. Martin, M. Ghadiri, U. Tüzün and B. Formisani, 2nd Symposium: Advances in Particulate Technology, Guildford, UK, 15-17 April 1991.

13) M.Ghadiri, J.A.S. Cleaver and R. Seaton, 1st European Symposium on Separation of Particles from Gases, PARTEC, Nuremberg, 19-21 April 1989.

14) C.M. Martin, M. Ghadiri, and U. Tüzün, 2nd World Congress Particle Technology, Kyoto, Japan, 19-22 Septmember 1990.

15) R.M. Nedderman, U. Tüzün and R.B. Thorpe, Powder Technol., 35(1983)69. 